\title{
Soluble ST2, Galectin-3 and clinical prognosis of patients with hypertrophic cardiomyopathy undergoing ventricular septal myectomy: a correlation analysis
}

\author{
Bangrong Song ${ }^{1}$, Bo Yao ${ }^{2}$, Haiming Dang ${ }^{1}$, Ran Dong ${ }^{1}$ \\ ${ }^{1}$ Department of Cardiac Surgery, Beijing Anzhen Hospital, Capital Medical University, Beijing 100029, China; ${ }^{2}$ Cardiology Section 2, Zibo City \\ Linzi District People's Hospital, Zibo 255400, China \\ Contributions: (I) Conception and design: B Song, R Dong; (II) Administrative support: R Dong; (III) Provision of study material or patients: H Dang; \\ (IV) Collection and assembly of data: H Dang; (V) Data analysis and interpretation: B Song, B Yao; (VI) Manuscript writing: All authors; (VII) Final \\ approval of manuscript: All authors. \\ Correspondence to: Ran Dong. Department of Cardiac Surgery, Beijing Anzhen Hospital, Capital Medical University, No. 2, Anzhen Road, Chaoyang \\ District, Beijing 100029, China. Email: jy199109101@163.com.
}

Background: Hypertrophic cardiomyopathy (HCM) is the most common chromosomal abnormal heart disease. The pathophysiological mechanism of HCM is complex. Several studies have suggested that the level of Soluble ST2 (sST2) may be a biomarker of chronic systolic heart failure, however, the role of sST2 in HCM remains unclear. So we performed this study to analyze the role of Soluble ST2 (sST2), Galectin-3 (Gal-3) and its correlations with clinical prognosis of patients with hypertrophic cardiomyopathy (HCM) undergoing ventricular septal myectomy.

Methods: HCM patients who underwent modified Morrow surgery in our hospital during June 2016-June 2018 were included. We divided the patients into different groups stratified by sST2 and Gal-3 level. Besides, we included volunteers without heart disease for medical examination as normal controls. Biochemical analyses were conducted to identify the biomarkers difference. The predictive value of sST2 and Gal-3 on all-cause mortality was evaluated with Cox regression analysis.

Results: A total of $125 \mathrm{HCM}$ patients were included in this present study. The sST2 and Gal-3 levels in $\mathrm{HCM}$ patients were significantly higher than that in control group (all $\mathrm{P}<0.001$ ); there were significant differences in the incidence of all-cause mortality for HCM patients stratified by the sST2 and Gal-3 level; Cox univariate regression survival analysis showed that the hypertension (HR $=1.19,95 \% \mathrm{CI}: 1.01-1.38$ ), maximum wall thickness ( $\mathrm{HR}=1.48,95 \% \mathrm{CI}: 1.04-1.98)$, Log sST2 (HR =1.02, 95\% CI: 1.01-1.05), Log Gal-3 (HR $=1.17,95 \% \mathrm{CI}: 1.09-1.32$ ) were the predictors for all-cause mortality in patients with HCM, and Cox multivariate risk regression showed that maximum wall thickness was the independent predictors of allcause mortality in patients with HCM (HR =1.63, 95\% CI: $1.35-1.97)$.

Conclusions: Even through sST2 and Gal-3 were not associated with clinical prognosis of patients with HCM undergoing ventricular septal myectomy, it may be involved in the progress of HCM, more studies are warranted to identify the potential mechanism and reverence value.

Keywords: Soluble ST2 (sST2); Galectin-3 (Gal-3); hypertrophic cardiomyopathy (HCM); correlation

Submitted Nov 17, 2019. Accepted for publication Dec 09, 2019.

doi: $10.21037 / \mathrm{cdt} .2020 .01 .04$

View this article at: http://dx.doi.org/10.21037/cdt.2020.01.04 


\section{Introduction}

Hypertrophic cardiomyopathy (HCM) is the most common chromosomal abnormal heart disease with an incidence of about 1:500 (1). It is the most common cause of sudden cardiac death in adolescents and athletes, and it's also a risk factor for heart failure and stroke $(2,3)$. The pathophysiological mechanism of HCM is complex with regards to that the genotype, clinical phenotype and prognosis are highly heterogeneous. The clinical features of HCM include asymmetric left atrial hypertrophy, abnormal myocardial energy metabolism, microcirculatory ischemia, cardiac muscle fibrosis, diastolic dysfunction and arrhythmia (4). The early detection of HCM is very important for the timely treatment intervention.

Recently, several studies $(5,6)$ have found that elevated levels of soluble ST2 (sST2) in patients with chronic systolic heart failure is a novel biomarker that enhances risk stratification. ST2 is a member of the interleukin-1 receptor family glycoprotein, which is widely involved in the process of myocardial remodeling (7). However, the role of sST2 in patients with HCM remains unclear. Galectin-3 (Gal-3) is a lectin secreted by activated macrophages and it's bound to beta galactose (8). It's been reported that Gal-3 is involved in multiple pathophysiological processes of heart failure, including myocardial fibrosis, inflammation, and myocardial remodeling $(9,10)$. A clinical study (11) has found that the Gal-3 level is an independent predictor of all-cause death and re-admission in patients with heart failure. Myocardial interstitial fibrosis and left ventricular diastolic function are impaired in patients with HCM (12), it's reasonable to assume that sST2 and Gal-3 may be participated in the progress of HCM. Therefore, we aimed to evaluate the roles of sST2 and Gal-3 in patients with HCM and to assess its correlation with clinical outcomes in patients with HCM undergoing ventricular septal myectomy.

\section{Methods}

\section{Ethical considerations}

This present study has been checked and approved by the ethics committee of Beijing Anzhen Hospital (No. 20161086).

\section{Participants}

HCM patients who underwent modified Morrow surgery in our hospital during June 2016-June 2018 were selected as study subjects. HCM was defined as significant left ventricular wall segmental hypertrophy, left ventricular end-diastolic maximum wall thickness $\geq 15 \mathrm{~mm}$, the other systemic or localized diseases that can cause ventricular hypertrophy should be excluded. The indications for ventricular septal myectomy were: the effects of drug treatment were undesired, left or lower left ventricular outflow/left ventricular pressure gradient $\geq 50 \mathrm{mmHg}$ at rest. The patients were excluded if the patients had history of cardiac surgery, myocardial ablation or severe liver and renal insufficiency. Our study was complied with the Declaration of Helsinki and was approved by the Ethics Committees of the participating hospitals. All patients provided written, informed consent.

Furthermore, we also selected 125 volunteers without heart disease for medical examination as normal controls with matched gender and age, we attempted to compare the differences of sST2 and Gal-3 levels in those two groups. All the patients were undergone five months period of follow-up, and all the related procedures were conducted in this present hospital.

\section{Biochemical analyses}

All blood samples were obtained in a fasting state on the morning of surgery day. Routine biochemistry was analyzed in the central laboratory of our hospital. The patient's total cholesterol (TC), triglyceride (TG), low-density lipoprotein cholesterol (LDL-C), NT-proBNP, and cardiac troponin I (cTnI) levels were detected and analyzed using commercially available assay (Henshe Diagnostics, Shanghai, China). The blood samples for the measurement of sST2 were collected in pyrogen-free tubes containing ethylenediaminetetraacetic acid and further measured by enzyme-linked immunosorbent assay with corresponding bioanalysis kits (x28408 and x29505, Xihui, China). All the procedures were conducted in comply with the related guidelines. The biochemical analyses were performed blindly.

\section{Echocardiography detection}

The transthoracic echocardiography was performed using Siemens N10 ultrasound system (German). The difference in pressure between the left ventricular outflow tract and the left ventricle was estimated using continuous Doppler ultrasound. The left atrial inner diameter, maximum wall thickness, left ventricular ejection fraction, left ventricular mass index $\left(\mathrm{g} / \mathrm{m}^{2}\right)$ 
were measured using methods recommended by the American Society of Echocardiography.

\section{Statistical analysis}

All statistical analyses were performed using SPSS 23.0 software. The measurement data were presented with mean \pm standard deviation $\left(\bar{x}_{ \pm} \mathrm{SD}\right)$. Comparisons between consecutive variables were performed using an independent sample $t$-test; the categorical variables were expressed as ratio (\%), and the comparisons between categorical variables were performed using a chi-square test. Survival curves were analyzed by Kaplan-Meier methods and plotted using GraphPad 6.0 software. Besides, to assess the predictors of clinical endpoints, the Cox proportional hazard regression model was utilized to calculate the hazard ratio (HR) and the $95 \%$ confidence interval (CI). Probability values (twosided) were considered significant at $\mathrm{P}<0.05$.

\section{Results}

\section{The characteristics of included HCM patients}

A total of $125 \mathrm{HCM}$ patients were included in this present study. Patients with HCM were divided into three groups based on the tertiles of sST2 level: low sST2 group $(<10.02 \mathrm{ng} / \mathrm{mL})$, medium sST2 group (10.02 to $15.98 \mathrm{ng} / \mathrm{mL}$ ), and high sST2 group $(>15.98 \mathrm{ng} / \mathrm{mL})$. No significant difference was detected in the age, gender, complicated diseases, drug use, blood laboratory results and echocardiographic analysis among the three sST2-stratified groups. The baseline characteristics of patients stratified by $\mathrm{sST} 2$ tertiles are presented in Table 1.

Meanwhile, patients with HCM were divided into three groups according to the tertiles of Gal-3 level: low Gal-3 group ( $<7.15 \mathrm{ng} / \mathrm{mL})$, medium Gal-3 group (7.15 to $9.04 \mathrm{ng} / \mathrm{mL}$ ), and high Gal-3 group (>9.04 ng/mL). No significant difference was detected in the age, gender, complicated diseases, drug use, blood laboratory results and echocardiographic analysis among the three Gal-3-stratified groups. The baseline characteristics of patients stratified by Gal-3 tertiles are presented in Table 2.

\section{The sST2 and Gal-3 differences between HCM and control group}

As Figure 1 presented, the sST2 and Gal-3 levels in HCM patients were significantly higher than that in control group (all $\mathrm{P}<0.001$ ).

\section{The incidence of all-cause mortality according to tertiles of sST2 and Gal-3}

The Kaplan-Meier survival analysis showed that there were significant differences in the incidence of all-cause mortality for HCM patients stratified by the sST2 and Gal-3 levels ( $\log$-rank $\mathrm{P}=0.032$ and 0.09 , respectively, Figure 2).

\section{Correlations between sST2 and clinical prognosis}

As Table 3 presented, Cox univariate regression survival analysis showed that the hypertension ( $\mathrm{HR}=1.19$, 95\% CI: 1.01-1.38), maximum wall thickness ( $\mathrm{HR}=1.48,95 \%$ CI: 1.04-1.98), Log sST2 (HR =1.02, 95\% CI: 1.01-1.05), Log Gal-3 (HR =1.17, 95\% CI: 1.09-1.32) were the predictors for all-cause mortality in patients with HCM. Therefore, we included the hypertension, maximum wall thickness, Log sST2, Log Gal-3 in the Cox multivariate risk regression model. The results showed that maximum wall thickness was the independent predictors of all-cause mortality in patients with HCM (HR $=1.63,95 \%$ CI: $1.35-1.97)$.

\section{Discussion}

As a kind of heart diseases, HCM is a serious threat to human life (13). For the HCM patients that medications may not effective in relieving symptoms, myocardial resection has been proven to be a safe and effective treatment, which can significantly improve the clinical symptoms and cardiac function $(14,15)$. Exploring the risk factors for clinical events in patients with HCM after ventricular septal myectomy will help to guide postoperative management $(16,17)$. The presence of myocardial fibrosis in HCM patients has been confirmed by reports of myocardial biopsy (18), and it has been found to be an independent predictor of major clinical endpoints in HCM patients (19). In recent years, it has been found $(20,21)$ that both ST2 and Gal-3 are involved in myocardial fibrosis and myocardial remodeling, which may be very promising new biomarkers for cardiovascular disease. This present study has demonstrated that the sST2 and Gal-3 levels enhance risk stratification and predict clinical events based on established risk factors. This study showed that the level of sST2 and Gal-3 are significantly elevated in HCM patients, even rough a follow-up of 12 months revealed no correlation 
Table 1 The demographic characteristics of 125 HOCM patients stratified by sST2 level

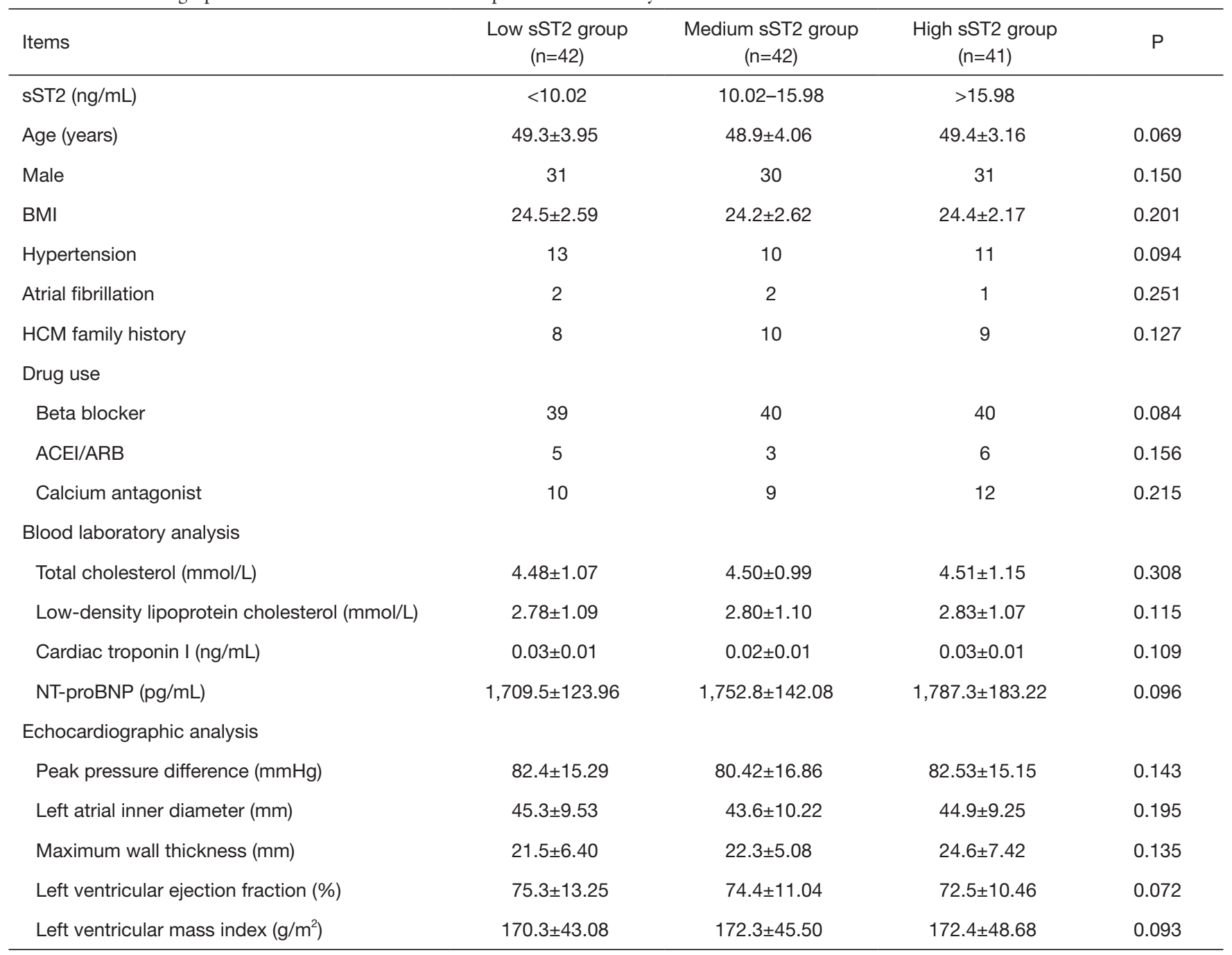

HOCM, hypertrophic obstructive cardiomyopathy; HCM, hypertrophic cardiomyopathy; ACEl, angiotensin-converting enzyme inhibitors; $\mathrm{ARB}$, angiotensin receptor blocker.

between sST2 and Gal-3 levels and clinical outcomes in patients with HCM after ventricular septal myectomy, and only maximum wall thickness is an independent predictor of the composite endpoint of all-cause death in patients with HCM after ventricular septal myocardial resection, the sST2 and Gal-3 levels may provide potentially valuable reference for HCM treatment.

sST2 is a member of the interleukin-1 receptor family that competes with the ST2L ligand in the cardiac IL$33 / \mathrm{ST} 2 \mathrm{~L}$ signaling pathway $(22,23)$. It inhibits cardio protection and anti-fibrosis of IL-33/ST2L signaling pathway by binding to IL-33 receptor. In recent years, many studies $(24,25)$ have found that sST2 in plasma is significantly elevated in patients with heart failure and is closely related to the prognosis of heart failure. Besides, as a new cardiac marker, sST2 has been reported to be involved in the activation of cardiac fibroblasts and myocardial fibrosis (26). Meanwhile, it's been reported that sST2 may be involved in the pathological process of myocardial fibrosis or myocardial remodeling mainly by regulating the degradation process of type I collagen (27). Previous studies have shown that for patients with chronic systolic heart failure, the baseline sST2 levels can increase the predictive value of clinical outcomes on the basis of natriuretic peptides, and continuous monitoring of ST2 levels can also predict patients' clinical outcomes (28). Still, the studies on 
Table 2 The demographic characteristics of 125 HOCM patients stratified by Gal-3 level

\begin{tabular}{|c|c|c|c|c|}
\hline Items & $\begin{array}{l}\text { Low Gal-3 group } \\
\qquad(n=39)\end{array}$ & $\begin{array}{l}\text { Medium Gal-3 group } \\
\qquad(n=44)\end{array}$ & $\begin{array}{l}\text { High Gal-3 group } \\
\qquad(\mathrm{n}=42)\end{array}$ & $\mathrm{P}$ \\
\hline Gal-3 (ng/mL) & $<7.15$ & $7.15-9.04$ & $>9.04$ & \\
\hline Age (years) & $49.4 \pm 2.46$ & $48.9 \pm 4.12$ & $49.1 \pm 4.86$ & 0.088 \\
\hline Male & 29 & 31 & 32 & 0.190 \\
\hline Atrial fibrillation & 1 & 2 & 2 & 0.101 \\
\hline HCM family history & 9 & 9 & 9 & 0.098 \\
\hline \multicolumn{5}{|l|}{ Drug use } \\
\hline Beta blocker & 38 & 41 & 40 & 0.144 \\
\hline \multicolumn{5}{|l|}{ Blood laboratory analysis } \\
\hline Total cholesterol (mmol/L) & $4.44 \pm 1.35$ & $4.62 \pm 0.83$ & $4.50 \pm 1.32$ & 0.948 \\
\hline Low-density lipoprotein cholesterol (mmol/L) & $2.73 \pm 1.14$ & $2.81 \pm 1.40$ & $2.50 \pm 1.46$ & 0.104 \\
\hline Cardiac troponin I (ng/mL) & $0.02 \pm 0.01$ & $0.03 \pm 0.01$ & $0.03 \pm 0.01$ & 0.091 \\
\hline NT-proBNP (pg/mL) & $1,732.4 \pm 130.56$ & $1,758.3 \pm 142.15$ & $1,788.4 \pm 184.36$ & 0.155 \\
\hline \multicolumn{5}{|l|}{ Echocardiographic analysis } \\
\hline Peak pressure difference $(\mathrm{mmHg})$ & $83.4 \pm 10.25$ & $84.3 \pm 14.46$ & $85.2 \pm 14.02$ & 0.194 \\
\hline
\end{tabular}

HOCM, hypertrophic obstructive cardiomyopathy; HCM, hypertrophic cardiomyopathy; ACEl, angiotensin-converting enzyme inhibitors; $\mathrm{ARB}$, angiotensin receptor blocker.

the role of sST2 in HCM patients are very few, mores studies are warranted in identifying the role sST2.

Gal-3 is a chimeric galectin that can be expressed in a variety of tissues and cells, and interacts with various ligands such as collagen, fibronectin, integrin and laminin in cells and matrix (29). It's been found that Gal-3 is widely active in inflammatory cells, Gal-3 binds to integrin to mediate cell adhesion (30), promote adhesion of macrophages and vascular endothelial cells (31), accelerate the proliferation and metastasis of smooth muscle cells (32), and accelerate the pathological changes of coronary plaques (33). Previous studies $(34,35)$ have shown that Galectin-3 is highly expressed in unstable regions of mouse and human arterial plaques, it's been well-believed that it relates to the progress of cardiovascular disease. It's been found that patients with heart failure with Gal-3 levels $>17.8 \mathrm{ng} / \mathrm{mL}$ were significantly more likely to be hospitalized for heart failure (36). In this present study, the sST2 and Gal-3 levels were not associated with clinical events in patients with HCM after ventricular septal myectomy, it may be explained that most HCM patients undergoing ventricular septal myectomy have a significant improvement in heart function, significant association results may be obtained if being analyzed in the populations of more severely-injurious population $(37,38)$. 


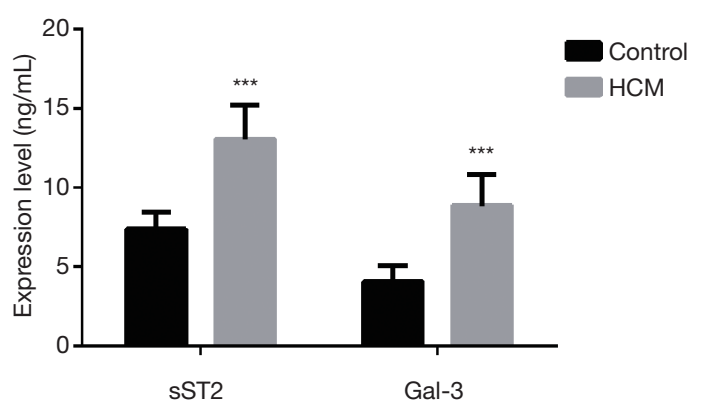

Figure 1 The sST2 and Gal-3 differences between HCM and Control group. ${ }^{* * *}, \mathrm{P}<0.001$ compared with control.

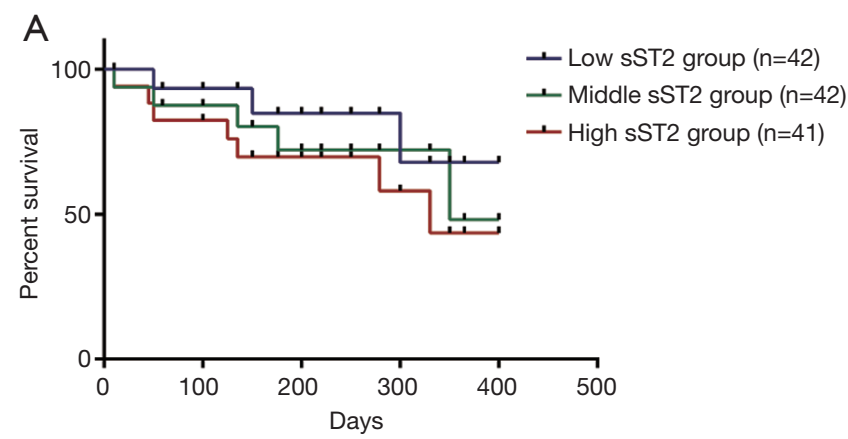

Several limitations should be concerned in this study. Firstly, the study sample is rather small, its statistical effects may be not enough to detect the correlation of sST2, Gal-3 levels and clinical prognosis of HCM patients, we are continuing to collect the related information to reach a larger sample size in the future. And HCM undergoing ventricular septal myomectomy is thought to be hypertrophic obstructive cardiomyopathy. The part of obstruction and the change of pressure gradient at obstructive site after ventricular septal myomectomy are important for prognosis. We attempted to include this data

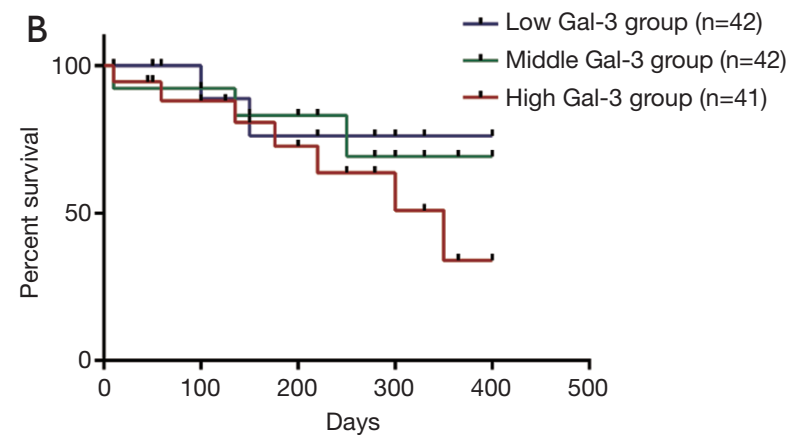

Figure 2 Kaplan-Meier plots depicting the incidence of all-cause mortality according to tertiles of sST2 and Gal-3. (A) The all-cause mortality according to tertiles of sST2; (B) Kaplan-Meier plots on the all-cause mortality according to tertiles of Gal-3.

Table 3 Cox risk regression analysis on the predictors of all-cause mortality

\begin{tabular}{|c|c|c|c|c|}
\hline Variables & \multicolumn{2}{|c|}{ Univariate regression } & \multicolumn{2}{|c|}{ Multivariate regression } \\
\hline Age & $1.04(1.00-1.08)$ & 0.184 & - & - \\
\hline Male & $1.74(0.96-2.45)$ & 0.130 & - & - \\
\hline Hypertension & $1.19(1.01-1.38)$ & 0.047 & $1.10(0.94-1.37)$ & 0.099 \\
\hline Peak pressure difference & $1.09(0.87-1.05)$ & 0.079 & - & - \\
\hline Maximum wall thickness & $1.48(1.04-1.98)$ & 0.042 & $1.63(1.35-1.97)$ & 0.015 \\
\hline Left ventricular ejection fraction & $1.90(0.82-1.05)$ & 0.195 & - & - \\
\hline Left ventricular mass index $\left(\mathrm{g} / \mathrm{m}^{2}\right)$ & $1.14(0.66-3.58)$ & 0.507 & - & - \\
\hline
\end{tabular}

HCM, hypertrophic cardiomyopathy; sST2, soluble ST2; Gal-3, Galectin-3. 
at the initial design, yet the most related data are missing, therefore, we cannot include this data. Secondly, due to the resource limitation, we only conducted one-year long follow-up for HCM patients, the longer all-cause mortality remains unclear, there is possibility that the sST2, Gal-3 level can predict the risk of longer period of all-cause mortality. Thirdly, we only detected the level of sST2, Gal-3 in one timepoint, the sST2, Gal-3 level after surgery should also be analyzed whenever possible, the changes of sST2, Gal-3 expression may be related to the effects of surgical treatment, which needs further investigation.

\section{Conclusions}

In conclusion, the results of this study indicated that sST2 and Gal-3 were not associated with all-cause mortality of patients with HCM undergoing ventricular septal myectomy, sST2 and Gal-3 provides limited clinical value for risk stratification in this population. However, our study do has some limitations, further studies are warranted to identify the role of sST2 and Gal-3 in cardiovascular diseases.

\section{Acknowledgments}

Funding: None.

\section{Footnote}

Conflicts of Interest: All authors have completed the ICMJE uniform disclosure form (available at http://dx.doi. org/10.21037/cdt.2020.01.04). The authors have no conflicts of interest to declare.

Ethical Statement: The authors are accountable for all aspects of the work in ensuring that questions related to the accuracy or integrity of any part of the work are appropriately investigated and resolved. This present study has been checked and approved by the ethics committee of Beijing Anzhen Hospital (No. 20161086).

Open Access Statement: This is an Open Access article distributed in accordance with the Creative Commons Attribution-NonCommercial-NoDerivs 4.0 International License (CC BY-NC-ND 4.0), which permits the noncommercial replication and distribution of the article with the strict proviso that no changes or edits are made and the original work is properly cited (including links to both the formal publication through the relevant DOI and the license).
See: https://creativecommons.org/licenses/by-nc-nd/4.0/.

\section{References}

1. Mosqueira D, Smith JGW, Bhagwan JR, et al. Modeling Hypertrophic Cardiomyopathy: Mechanistic Insights and Pharmacological Intervention. Trends Mol Med 2019;25:775-90.

2. Cao Y, Zhang PY. Review of recent advances in the management of hypertrophic cardiomyopathy. Eur Rev Med Pharmacol Sci 2017;21:5207-10.

3. Sedaghat-Hamedani F, Kayvanpour E, Tugrul OF, et al. Clinical outcomes associated with sarcomere mutations in hypertrophic cardiomyopathy: a meta-analysis on 7675 individuals. Clin Res Cardiol 2018;107:30-41.

4. Guo X, Fan C, Tian L, et al. The clinical features, outcomes and genetic characteristics of hypertrophic cardiomyopathy patients with severe right ventricular hypertrophy. PLoS One 2017;12:e0174118.

5. Matilla L, Ibarrola J, Arrieta V, et al. Soluble ST2 promotes oxidative stress and inflammation in cardiac fibroblasts: an in vitro and in vivo study in aortic stenosis. Clin Sci (Lond) 2019;133:1537-48.

6. Berezin AE, Kremzer AA, Samura TA, et al. Altered signature of apoptotic endothelial cell-derived micro vesicles predicts chronic heart failure phenotypes. Biomark Med 2019;13:737-50.

7. You H, Jiang W, Jiao M, et al. Association of Soluble ST2 Serum Levels With Outcomes in Pediatric Dilated Cardiomyopathy. Can J Cardiol 2019;35:727-35.

8. Gehlken C, Suthahar N, Meijers WC, et al. Galectin-3 in Heart Failure: An Update of the Last 3 Years. Heart Fail Clin 2018;14:75-92.

9. Suthahar N, Meijers WC, Sillje HHW, et al. Galectin-3 Activation and Inhibition in Heart Failure and Cardiovascular Disease: An Update. Theranostics 2018;8:593-609.

10. Málek F. Impact of galectin 3 as myofibrosis marker in clinical cardiology. Vnitr Lek 2014;60:327-30.

11. van Vark LC, Lesman-Leegte I, Baart SJ, et al. Prognostic Value of Serial Galectin-3 Measurements in Patients With Acute Heart Failure. J Am Heart Assoc 2017. doi: 10.1161/ JAHA.116.003700.

12. Sarhene M, Wang Y, Wei J, et al. Biomarkers in heart failure: the past, current and future. Heart Fail Rev 2019;24:867-903.

13. Choudhry S, Puri K, Denfield SW. An Update on Pediatric Cardiomyopathy. Curr Treat Options Cardiovasc Med 2019;21:36.

14. Batzner A, Schafers HJ, Borisov KV, et al. Hypertrophic 
Obstructive Cardiomyopathy. Dtsch Arztebl Int 2019;116:47-53.

15. Hodges K, Rivas CG, Aguilera J, et al. Surgical management of left ventricular outflow tract obstruction in a specialized hypertrophic obstructive cardiomyopathy center. J Thorac Cardiovasc Surg 2019;157:2289-99.

16. Norrish G, Cantarutti N, Pissaridou E, et al. Risk factors for sudden cardiac death in childhood hypertrophic cardiomyopathy: A systematic review and meta-analysis. Eur J Prev Cardiol 2017;24:1220-30.

17. Makavos G, Kappaairis C, Tselegkidi ME, et al. Hypertrophic cardiomyopathy: an updated review on diagnosis, prognosis, and treatment. Heart Fail Rev 2019;24:439-59.

18. Marian AJ, Braunwald E. Hypertrophic Cardiomyopathy: Genetics, Pathogenesis, Clinical Manifestations, Diagnosis, and Therapy. Circ Res 2017;121:749-70.

19. Briasoulis A, Mallikethi-Reddy S, Palla M, et al. Myocardial fibrosis on cardiac magnetic resonance and cardiac outcomes in hypertrophic cardiomyopathy: a metaanalysis. Heart 2015;101:1406-11.

20. Tuegel C, Katz R, Alam M, et al. GDF-15, Galectin 3, Soluble ST2, and Risk of Mortality and Cardiovascular Events in CKD. Am J Kidney Dis 2018;72:519-28.

21. Lepojärvi ES, Piira OP, Paakko E, et al. Serum PINP, PIIINP, galectin-3, and ST2 as surrogates of myocardial fibrosis and echocardiographic left venticular diastolic filling properties. Front Physiol 2015;6:200.

22. Gao Y, Zhang MY, Wang T, et al. IL-33/ST2L Signaling Provides Neuroprotection Through Inhibiting Autophagy, Endoplasmic Reticulum Stress, and Apoptosis in a Mouse Model of Traumatic Brain Injury. Front Cell Neurosci 2018;12:95.

23. Asensio-Lopez MC, Lax A, Fernandez Del Palacio MJ, et al. Yin-Yang 1 transcription factor modulates ST2 expression during adverse cardiac remodeling post-myocardial infarction. J Mol Cell Cardiol 2019;130:216-33.

24. Dieplinger B, Mueller T. Soluble ST2 in heart failure. Clin Chim Acta 2015;443:57-70.

25. Sinning C, Kempf T, Schwarzl M, et al. Biomarkers for characterization of heart failure - Distinction of heart failure with preserved and reduced ejection fraction. Int J Cardiol 2017;227:272-7.

26. Sanada S, Hakuno D, Higgins LJ, et al. IL-33 and ST2 comprise a critical biomechanically induced and cardioprotective signaling system. J Clin Invest 2007;117:1538-49.

27. Martínez-Martínez E, Miana M, Jurado-Lopez R, et al. A role for soluble ST2 in vascular remodeling associated with obesity in rats. PLoS One 2013;8:e79176.
28. Greenway SC. The Continuation of Trickle-Down Medicine: Soluble Suppression of Tumorigenicity-2 (sST2) in Pediatric Heart Failure. Can J Cardiol 2019;35:692-3.

29. Di Tano G, Caretta G, De Maria R, et al. Galectin-3 and outcomes after anterior-wall myocardial infarction treated by primary percutaneous coronary intervention. Biomark Med 2018;12:21-6.

30. Yang EH, Rode J, Howlader MA, et al. Galectin-3 alters the lateral mobility and clustering of beta1-integrin receptors. PLoS One 2017;12:e0184378.

31. Ibarrola J, Martinez-Martinez E, Sadaba JR, et al. Beneficial Effects of Galectin-3 Blockade in Vascular and Aortic Valve Alterations in an Experimental Pressure Overload Model. Int J Mol Sci 2017. doi: 10.3390/ijms18081664.

32. Barman SA, Li X, Haigh S, et al. Galectin-3 is expressed in vascular smooth muscle cells and promotes pulmonary hypertension through changes in proliferation, apoptosis, and fibrosis. Am J Physiol Lung Cell Mol Physiol 2019;316:L784-L797.

33. MacKinnon AC, Liu X, Hadoke PW, et al. Inhibition of galectin-3 reduces atherosclerosis in apolipoprotein E-deficient mice. Glycobiology 2013;23:654-63.

34. Du XJ, Zhao WB, Nguyen MN, et al. beta-Adrenoceptor activation affects galectin-3 as a biomarker and therapeutic target in heart disease. Br J Pharmacol 2019;176:2449-64.

35. Souza BSF, Silva DN, Carvalho RH, et al. Association of Cardiac Galectin-3 Expression, Myocarditis, and Fibrosis in Chronic Chagas Disease Cardiomyopathy. Am J Pathol 2017;187:1134-46.

36. Wang CH, Cheng ML, Liu MH, et al. Metabolic profile provides prognostic value better than galectin-3 in patients with heart failure. J Cardiol 2017;70:92-8.

37. Lax A, Sanchez-Mas J, Asensio-Lopez MC, Fernandez-Del Palacio MJ, et al. Mineralocorticoid receptor antagonists modulate galectin-3 and interleukin-33/ST2 signaling in left ventricular systolic dysfunction after acute myocardial infarction. JACC Heart Fail 2015;3:50-8.

38. Bayes-Genis A, de Antonio M, Vila J, et al. Head-to-head comparison of 2 myocardial fibrosis biomarkers for longterm heart failure risk stratification: ST2 versus galectin-3. J Am Coll Cardiol 2014;63:158-66.

Cite this article as: Song B, Yao B, Dang H, Dong R. Soluble ST2, Galectin-3 and clinical prognosis of patients with hypertrophic cardiomyopathy undergoing ventricular septal myectomy: a correlation analysis. Cardiovasc Diagn Ther 2020;10(2):145-152. doi: 10.21037/cdt.2020.01.04 